\title{
Dynamic Characteristics of Overhead Transmission Lines following Ice Shedding Based on Lagrange Equation
}

\author{
Fan Peng ${ }^{1, a}$ \\ ${ }^{1}$ Shool of Control and Computer Engineering, North China Electric Power University, \\ Beijing,102206, China \\ aemail:18800166696 @163.com
}

Keywords: Overhead Transmission Lines; Ice Shedding; Lagrange Equation;Dynamic Characteristics

\begin{abstract}
Transmission line icing coating will lead to ice shedding when ambient temperature rises or the wind increases. It can cause serious security threat to the structure of the transmission line system. In the paper, based on the Lagrange Equation, the analytical dynamics equations of transmission lines ice shedding are deduced in theory. In these equations we comprehensively consider the factors affecting the dynamic characteristics of the ice shedding and do quantitative analysis. Taking a certain transmission line as an example, considering three kinds of working condition of ice shedding , our system analyzes the effect of ice shedding position and ice shedding proportion on the dynamic characteristics of a transmission line. Therefore we obtain a deeper understanding of problems of transmission lines ice shedding. The paper puts forward the method of important engineering value that could be used to direct line maintenance and design and guarantee the safe operation of the transmission line.
\end{abstract}

\section{Introduction}

Research on transmission line ice shedding mainly contains method of field observation, model experiment and numerical simulation. In the first way, Morgan and Swift have measured $132 \mathrm{kV}$ transmission 5-span line, using hanging heavy to simulate ice coating load. In model experiment, Jamalendding et al from Canada created the artificial climate and do static load test of ice shedding on $3.22 \mathrm{~m}$ long line in laboratory, to simulate many different ice shedding situation [1]. But the test, restricted by funding and site conditions, hasn't got good results. In the numerical simulation, Jamalendding, Kalman, McClure [2-4] and others tested on ice shedding of the line by sudden loading with a commercial software ADINA. They got static and dynamic responses of ice shedding of the line, and compared the numerical solutions with experimental results proving the reliability of the numerical methods. Zhou Di did investigation on ice-shedding induced by wind for iced lines and obtained calculation methods and formulas of maximum Shear force of the line and maximum line torsion angle [5], Liu Heyun studied the ice shedding mechanism and made the comparison of three different mechanisms [6], Hou Lei analyzed the nonlinear dynamic process of line ice shedding, established the model of ice shedding of three degrees of freedom , multi-span. By the central difference explicit scheme based on the dynamic equation, dynamic simulation has been gotten [7].

From previous studies, the finite element method has been used in many researches. However, the ice shedding, including uneven ice shedding, the whole span ice shedding, and nonsynchonous ice shedding, may be affected by the temperature, span, ice thickness et al, Building finite element model to analyze ice shedding is complex and time-consuming, its solution process may encounter some unexpected problems, making it difficult to summarize common problems and regular conclusions. In view of this, the paper establishes kinetic equations for line ice shedding based on Lagrange Equation and solved analytically. The paper studies the key indicators of the line like vibration displacement and tension, which are affected by ice shedding position, the amount of cover ice and shedding ice and other factors, so as to provide a simple and practical method of analysis for line ice shedding. 


\section{Mathematical Model for Ice Shedding}

\subsection{Fundamental Assumption}

Ice shedding is influenced by many factors, ice shedding model is also complex. Directly building ice shedding model reflecting the whole transmission line system is difficult. To simplify the problem, the paper is based on the following assumption.

1 ) The tower is fixed, there is no deformation during ice shedding; 2) Ignore the influence of ice shedding to span and interaction between span and span; 3) Ice shedding does not cause other icing line to shed; 4) Ice shedding occurs only in a certain position of a wire (including whole ice shedding), ice shedding in many place at the same time won't happen; 5) Ice shedding only cause horizontal single mode vibration.

Shown in Figure 1, before icing mass per unit length of bare wire is $\rho 0$, the initial horizontal tension is $\mathrm{H} 0$, tensile stiffness is EA0, lateral damping ratio is $\xi 0$; mass per unit length after full icing is $\rho 1$, stretching stiffness EA1, the lateral damping ratio $\xi 1$, horizontal tension H1.Line span is $\mathrm{L}$, ice shedding position is between the abscissa $\mathrm{x} 0$ to $\mathrm{x} 1$. The initial position of line is defined as the equilibrium position of the icing line only withstanding dead weight, shown in dashed lines. When the line is in the initial position, the sudden release of the line (partly icing) leads to the vibration response, namely the shed response of ice shedding.

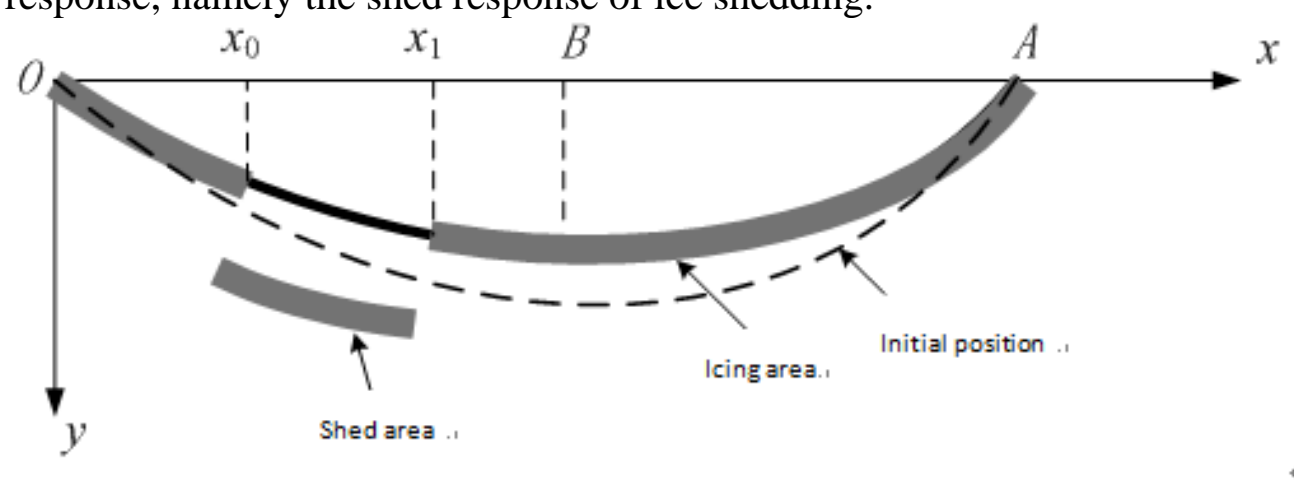

Fig.1. ice shedding schematic diagram

\subsection{Establishment of Motion Equations}

\section{2 .1Lagrange Equation}

With Langrage Equation using virtual coordinate method, dynamic relationship between objects is shown .The mathematical description is

$$
\frac{d}{d t}\left(\frac{\partial L}{\partial \dot{q}}\right)-\frac{\partial L}{\partial q}+\frac{\partial R}{\partial \dot{q}}=F
$$

Where, $L=T-V$, $T$ represents kinetic energy under a virtual coordinate ,and $\mathrm{V}$ represents potential energy under a virtual coordinate, and $\mathrm{R}$ represents dissipation function, and $\mathrm{Q}$ represents generalized displacement, and F represents the corresponding generalized force of non-conservative force.

We regard ice shedding as synchronized movement, so the vibration response of the ice shedding line is expressed as

$$
y(x, t)=Y(x) \cdot V(t)
$$

Where, $\mathrm{Y}(\mathrm{x})$ represents the shape function of the line in space, namely the vibration modes; $\mathrm{V}(\mathrm{t})$ denotes the motion displacement of each point on the line relative to the equilibrium position at time $t$, that is generalized displacement. Without loss of generality, our paper selects the sine function that satisfies the geometric boundary conditions as the vibration mode of the line, fixed at both ends during ice shedding, its expression is

$$
Y(x)=\sin \left(\frac{n \pi}{L} x\right)
$$

Thus the formula (2) can be expressed as 


$$
y(x, t)=\sin \left(\frac{n \pi}{L} x\right) \cdot V(t)
$$

\subsubsection{Kinetic Energy in Ice Shedding}

Kinetic energy on the line includes the iced and the non -iced segments, the expression is:

$$
T=\frac{1}{2}\left(\int_{0}^{x_{0}} \rho_{1} \sin ^{2}\left(\frac{n \pi}{L} x\right) d x+\frac{1}{2} \int_{x_{0}}^{x_{1}} \rho_{0} \sin ^{2}\left(\frac{n \pi}{L} x\right) d x+\frac{1}{2} \int_{x_{1}}^{L} \rho_{1} \sin ^{2}\left(\frac{n \pi}{L} x\right) d x\right) \cdot \dot{V}^{2}
$$

\subsubsection{Potential Energy in Ice Shedding}

Strain energy of the line is elastic strain energy, including the initial stress-induced strain energy and the strain energy generated by vibration. Strain energy is calculated based on the availability of the total strain energy of the wire is calculated according to the formula for strain energy

$$
V_{e}=\int_{0}^{L} H_{2} \cdot \varepsilon_{s} d x+\frac{1}{2} \int_{0}^{x_{0}} E A_{1} \cdot \varepsilon_{s 1}^{2} d x+\frac{1}{2} \int_{x_{0}}^{x_{1}} E A_{0} \cdot \varepsilon_{s 2}^{2} d x+\frac{1}{2} \int_{x_{1}}^{L} E A_{1} \cdot \varepsilon_{s 3}^{2} d x
$$

Where, $\mathrm{H}_{2}$ is the horizontal tension in the state of statics; $\varepsilon_{s 1}, \varepsilon_{s 2}$ and $\varepsilon_{s 3}$ correspond the dependent variable of three segments

Setting the gravitational potential energy at the coordinate origin to be zero, the gravitational potential energy of the ice shedding line is

$$
V_{g}=-\left[\rho_{0}\left(x_{1}-x_{0}\right)+\rho_{1}\left(L-x_{1}+x_{0}\right)\right] g \int_{0}^{L}\left[Y_{0}(x)+y(x, t)\right] d x
$$

The total potential energy of the line consist of the strain energy and gravitational potential energy.

\subsubsection{Dissipation Function}

The mechanical energy dissipation caused by dissipation force is the dissipation function, where the dissipation force is the linear viscous damp. By definition you can get dissipation function is:

$$
\begin{aligned}
R & =\frac{1}{2} \int_{0}^{L} c \cdot\left(\frac{\partial y}{\partial t}\right)^{2} d x=\frac{1}{2}\left[\int_{0}^{x_{0}} c_{1} \sin ^{2}\left(\frac{n \pi}{L} x\right) d x+\int_{x_{0}}^{x_{1}} C_{0} \sin ^{2}\left(\frac{n \pi}{L} x\right) d x+\int_{x 1}^{L} C_{1} \sin ^{2}\left(\frac{n \pi}{L} x\right) d x\right] \cdot \dot{V}^{2} \\
& =\frac{1}{2}\left(C_{1}+C_{2}+C_{3}\right) \cdot \dot{V}^{2}=\frac{1}{2} C \cdot \dot{V}^{2}
\end{aligned}
$$

In formula above , C1、C2and C3 represent the line damping matrix. Putting equation (4) - (8) into equation (1) Lagrange equation of ice shedding with a dissipation function will be got.

\section{Numerical Simulation Studying and Analysis}

In this paper, taking Runge - Kutta method to solve, selecting a typical set of data as an example, we get the line's specific parameters as shown in Table 1.

\section{Table 1 The parameters of line in ice shedding}

Span $\mathrm{L} / \mathrm{m}$

Mass per unit length of bare wire $\rho_{0} / \mathrm{kg} / \mathrm{m}$

Mass per unit length of iced wire $\rho 1 / \mathrm{kg} / \mathrm{m}$

Tensile stiffness of bare wireEA $\mathrm{A}_{0} / \mathrm{N}$

Tensile stiffness of iced wire $\mathrm{EA}_{1} / \mathrm{N}$

Bare wire transverse damping ratio $\xi_{0}$

Iced wire transverse damping ratio $\xi_{1}$

Bare wire horizontal tension $\mathrm{H}_{0} / \mathrm{kN}$ 
In order to compare the dynamic response characteristic in ice shedding in different position and proportion, the paper deal with 8 working conditions shown in Table 2.

Table 2 Running condition of lines in ice shedding

\begin{tabular}{clll}
\hline Working Condition & Position & Proportion & Ice-amount 1p1 \\
\hline Working condition 1 & $20 \sim 44$ & $10 \%$ & 4.3 \\
Working condition 2 & $70 \sim 94$ & $10 \%$ & 4.3 \\
Working condition 3 & $108 \sim 132$ & $10 \%$ & 4.3 \\
Working condition 4 & $20 \sim 92$ & $30 \%$ & 4.3 \\
Working condition5 & $84 \sim 156$ & $30 \%$ & 4.3 \\
Working condition 6 & $20 \sim 140$ & $50 \%$ & 4.3 \\
Working condition 7 & $60 \sim 180$ & $50 \%$ & 4.3 \\
Working condition 8 & $0 \sim 240$ & $100 \%$ & 4.3 \\
\hline
\end{tabular}

Figure 2 shows the displacement response of the line after ice shedding in $1 \mathrm{~s}, 5 \mathrm{~s}, 10 \mathrm{~s}, 50 \mathrm{~s}, 80 \mathrm{~s}$, 150s, 250s. The zero point of y-coordinate is the static equilibrium position of the line.

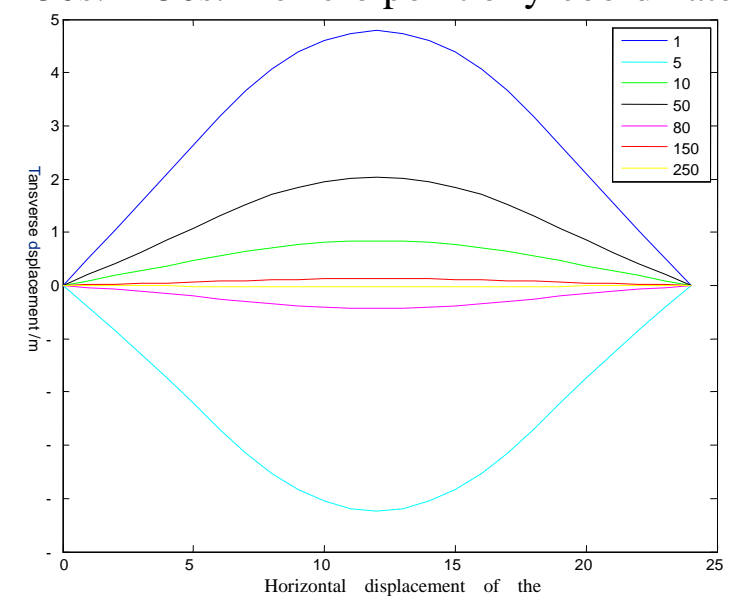

Fig.2. Displacement at different moment during ice shedding

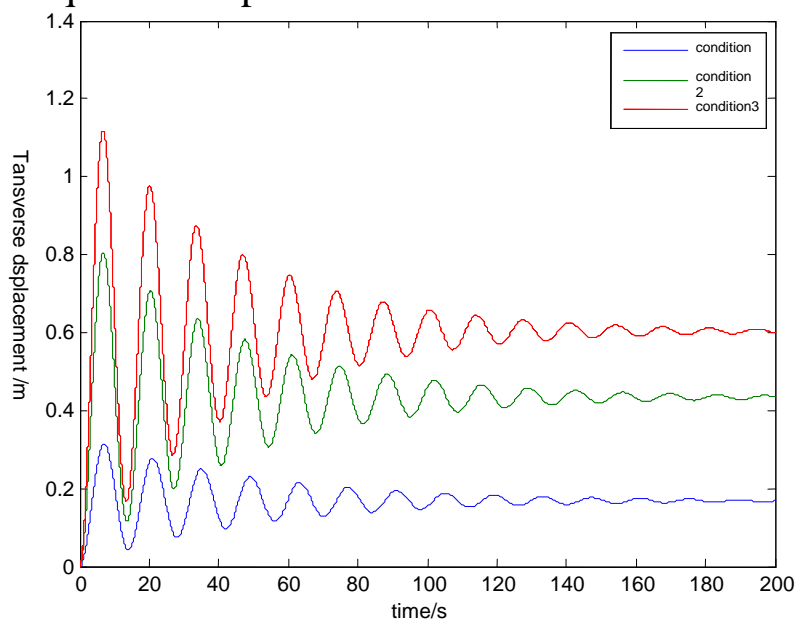

Fig.3.Displacement-time for different ice shedding position

\subsection{Analysis of Ice Shedding Position}

The paper select working condition 1 to 3to study the influence of different ice shedding position on the vibration response, Three conditions have the same ice shedding proportion of $10 \%$, but the positions of ice shedding are located at the extreme left, left and middle. B is the midpoint of the line. And the horizontal ice-shedding displacement response and the change in the horizontal tension of B are shown in Figure 3 and Figure 4.In figure 3, the zero point is at the initial position of the iced line.

From the displacement response in Figure 3, it can be seen that in the three conditions, before ice shedding, the midpoint is at the zero point. The maximum displacement responses are all present in a relatively short time after ice shedding. As is shown in Figure 3, after 6.6s, all the former three conditions have reached the maximum, respectively $0.3132 \mathrm{~m}, 0.8033 \mathrm{~m}, 1.118 \mathrm{~m}$, the closer the ice shedding position is to the middle position, the greater the vibration displacement is .On condition 1 , it keeps stable for a relatively short time in the last .in about 150s the peak value has been less than $0.01 \mathrm{~m}$, and the amplitude has been basically stable. In condition 2 and 3 it keep stable for relatively longer time, and with the move to the middle position, settling time is relatively increased. This is in good agreement with the actual situation. The midpoint of the line in static equilibrium position is 
relatively farthest away from the midpoint of the iced equilibrium position, and the gained elastic potential energy is the greatest. So after ice shedding in the Middle position, the vibration amplitude is great and the final system stability need more time.

As can be seen from Figure 4, with the ice shedding position moving close to the middle position, fluctuations in the amplitude of tension keep growing. Under condition 3, the maximum tension is $49 \mathrm{kN}$, minimum tension is $41.6 \mathrm{kN}$, reaching the maximum change in amplitude of tension. What's more, in the first 50s, the line is always in large low-frequency fluctuations process, this alternating force will be directly applied to the structure connected with the tower, bringing direct destruction to it.

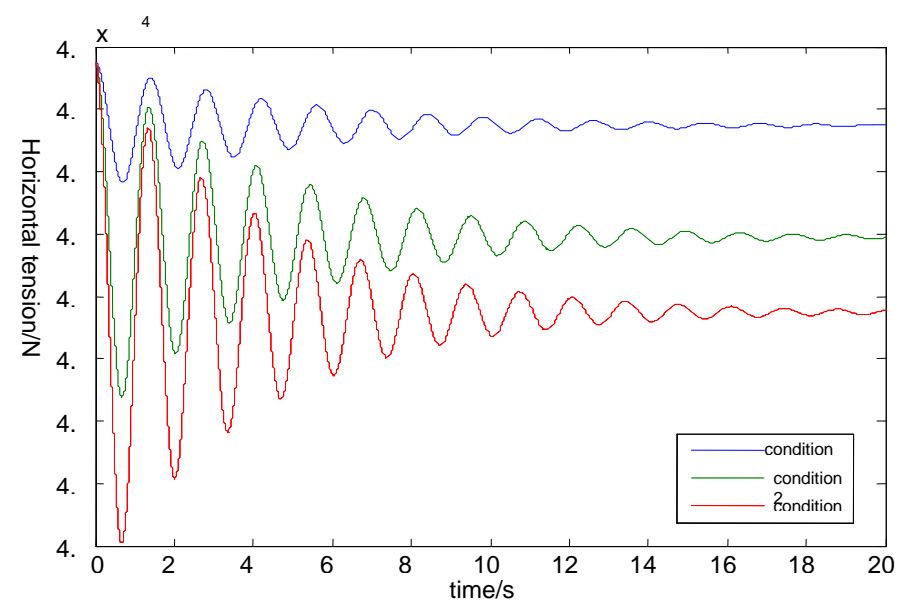

Fig.4. Tension-time for different ice shedding position

\subsection{Analysis of Ice Shedding Proportion}

The paper selects conditions 3, 5, 7and 8 to analyze the impact of different ice shedding proportion on vibration response. For these four conditions ice shedding all occurs in the middle of the line, in different ice shedding proportion of $10 \%, 30 \%, 50 \%$ and $100 \%$. Changes in horizontal tension and displacement response of the line after transverse ice shedding are shown in Figure 5 and Figure 6.In Figure 5, the zero point is at the initial position of the iced line.

As can be seen from the displacement response in Figure 5, the maximum displacement responses of four conditions probably occur in about 6.1s after ice shedding. As the ice shedding proportion increases, the amplitude of the line becomes higher. The maximum displacement of the line, for $100 \%$ ice shedding, reaches $6.111 \mathrm{~m}$. After 220s working condition 3, 5 have been basically stable, while the working conditions 7,8 still have not stabilized, especially under $100 \%$ ice shedding. In working condition 8 , after 220 s the peak value is still about $0.2 \mathrm{~m}$. As is shown in the figure, with an increase in ice shedding proportion, both oscillation amplitude and stability time are gradually increasing, and the change in magnitude is particularly evident. This law is similar to the one above .So when ice shedding occurs, the more the ice fall off , the more middle the position is, the greater the harm can be .

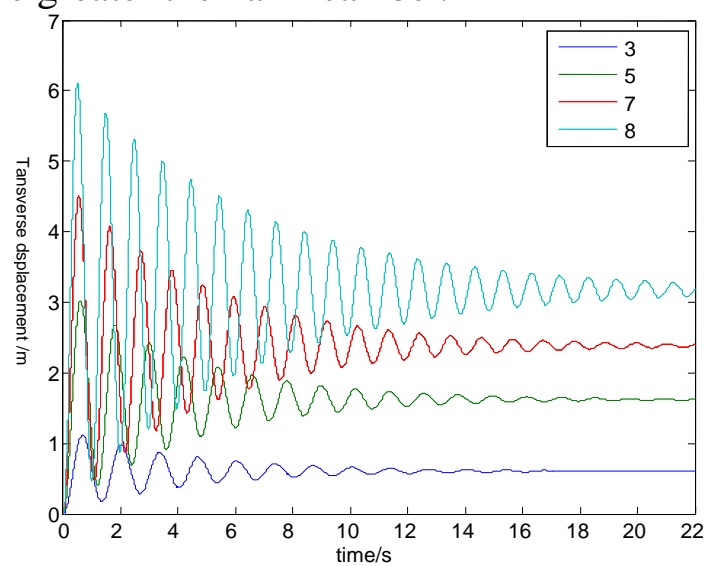

Fig.5.Displacement -time for different ice shedding proportion

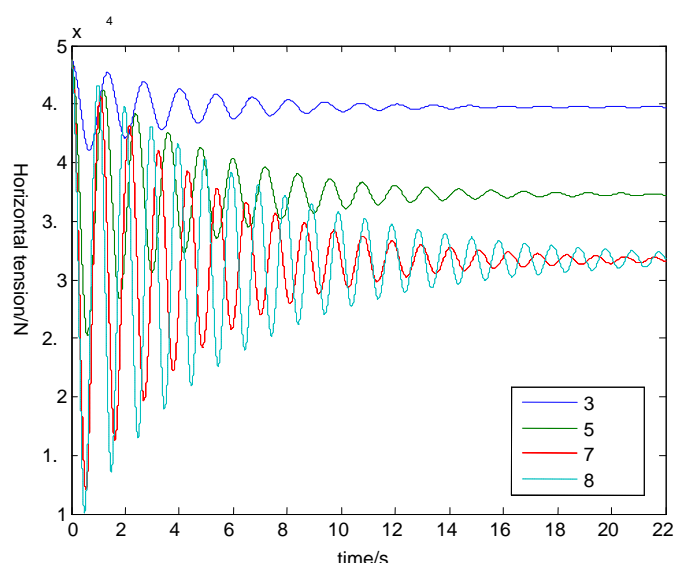

Fig.6. Tension-time for different ice shedding proportion 
It can be seen in Figure 6, from working condition 3 to 7 all the tension have the same rule: with the increase in ice shedding proportion, the maximum peak value of vibration and the stable value on amplitude go on.But when the ice shedding proportion is greater than $50 \%$, its growth rate is not obvious. It can be seen in working condition 8 , the proportion of $100 \%$, the difference between $50 \%$ and $100 \%$ is little ,both the magnitude of the horizontal tension are finally in stable condition in about $31.7 \mathrm{kN}$. Compared to different ice shedding position, ice shedding proportion of $50 \%$, the biggest change in tension reaches about $40 \mathrm{kN}$, and lasts about one minute, the tension of such a large oscillation and long period on the line will have a very serious impact on the tower.

\section{Conclusion}

The paper researches on the methods to establish the kinematic model of the line in ice shedding based on the Lagrange equation, and uses Runge - Kutta method to work out numerical solution of differential equations for ice shedding. Then it simulates the displacement response and tension changes of the wire in ice shedding. The paper respectively discussed the impact of ice shedding position and proportion. It should be noted that under natural conditions, the actual ice shedding process may occur at not only a single place. Since ice shedding in a certain segment may cause the ice load to spread in several directions at the same time, further ice shedding may occur in other segment. Continuous "initial displacement" and "initial velocity" in different places will generate a superposition of vibration impact in other segment. Theoretical model and solving method established in this paper and it lays a good foundation for the further study of complex conditions of ice shedding.

\section{References}

[1]A. Jamaledding, et al. Weigh-dropping Simulation of ice-shedding effects on an overhead transmission line model. Proceedings of 7th IWAIS (International workshop on Atmospheric Icing of Structures), Canada. $1996: 44-48$.

[2]A. Jamaledding. Simulation of ice-shedding on electrical transmission line using ADINA. Computers \& Structures, 1993, 47(4/5):523-536.

[3]T. Kalman, M. Farzaneh, G. MeClure. Numerical analysis of the dynamic effects of shock-load-induced ice shedding on overhead ground wires. Computers \& Structures, 2007, 85(7-8):375-38.

[4]M. RoshanFe and G...MeClure. Numerical modeling of the dynamic response of ice-shedding on electrical transmission lines. Atmospheric Research, 1998, 46(l-2):l-11.

[5] Liu Heyun. Research on Icing Coating and Icing Shedding Mechanism for Overhead Conductors [D] Wuhan: Huazhong University of Science and Technology, 2001.

[6] Wang Wenliang, Zhang, Luo Weide Structural Dynamics [M] Shanghai: Fudan University Press, 1993.

[7] Ke Sheng, Tang Guojin Analysis of Nonlinear Vibration Response under Suspension Chain [J] Vibration and Shock, 2003 (2): 69-72. 